\title{
Pathology of thymoma-where are we today?
}

\author{
Irshad Nabi Soomro \\ Department of Histopathology, Nottingham University Hospital, Nottingham, UK \\ Correspondence to: Irshad Nabi Soomro. Consultant Histopathologist and Honorary Clinical Associate Professor, Department of Histopathology, \\ Nottingham University Hospitals, Hucknall Road, Nottingham NG5 1PB, UK. Email: Irshad.soomro@nuh.nhs.uk.
}

\begin{abstract}
Thymoma is the commonest epithelial neoplasm arising from thymus gland. Tumour is slow growing and in the absence of metastasis, surgery is the treatment of choice. Complete resection and bland morphology are important prognostic features. However, a significant proportion of these tumours tend to recur. These recurrent tumours, advanced thymomas and thymic carcinomas require platinum-based combination chemotherapy and radiotherapy. Efforts are being made to explore additional treatment modalities to control disease with the aim of improving survival. Number of thymoma cases worldwide is small in comparison to lung cancers. As a result, fewer studies have been carried out to enhance our understanding of molecular events responsible for the initiation, maintenance, and progression of thymomas. Inspite of this there are advances in understanding the pathology of thymic epithelial neoplasms including genetics, PD-L1 and molecular testing which has bearing on the prognosis, post-surgical management, and testing algorithm. Similar to pulmonary pathology, thymic epithelial tumours will require adequate tumour sampling to carry out ancillary testing. Mutational analytical tests include $E G F R, R A S$, $B R A F, R E T, A K T 1, P I K 3 C A$ and T53 genes. If adequate sample is available (upto100 cells), PD-L1 testing should be considered for immunotherapy in recurrent/ advanced thymomas and thymic carcinomas. This list is likely to expand in future with increasing emphasis on molecular testing to support treatment with newer therapies.
\end{abstract}

Keywords: Thymoma; genetics; personalized medicine

Submitted Dec 11, 2019. Accepted for publication Jun 10, 2020.

doi: $10.21037 /$ jtd-2019-thym-04

View this article at: http://dx.doi.org/10.21037/jtd-2019-thym-04

\section{Introduction}

Significant progress has been made in understanding the biology, genetics, assessment of prognosis and personalized medicine for thymic epithelial neoplasms especially thymomas. This article is an effort to capture some of these developments in order to enhance our understanding of biology and subsequent management of these tumours.

\section{Thymus gland}

The most common neoplasm arising from the thymus gland is thymoma. This is the most common anterior mediastinal neoplasm. This tumour can also occur in other sites which include lung, pleura, and neck region. It is seen after the age of 40 and approximately $33 \%$ are invasive. Thymomas are associated with myasthenia gravis and autoimmune diseases such as red cell aplasia, hypogammaglobinaemia, polymyositis, rheumatoid arthritis, Sjogren's syndrome, thyroiditis, graft versus host like disease and pancytopenia.

\section{Histological features of thymoma}

Epithelial cells of variable morphology including polygonal, stellate and spindle shapes are seen. These are mixed with mainly $\mathrm{T}$ lymphocytes. The epithelial cells are positive for CK7, CK5/6, P63, P40, CD205, Foxn1 and PAX8. Most of the T lymphocytes stain for TdT, CD99, CD1a exhibiting immature phenotype. There is a population of S-100 protein and CD1a positive cells, interdigitating reticulum cells. Asteroid cells staining for B-cell marker CD20 and myoid cells staining for desmin and myoglobin are also present. Most thymomas have cytologically bland epithelial and non-neoplastic lymphocyte components. Prognostic factors are high stage, positive margins, and podoplanin over expression which may predict lymph node metastasis (1). 
Thymic squamous cell carcinomas can be distinguished from type $\mathrm{B} 3$ thymomas on the basis of morphology and by the presence of CD5, CEA, MUC-1, CD117, CD1a, TdT, and GLUT-1 immunostaining. There is higher expression of P53, Bcl-2 and p16 in carcinomas than thymomas (2).

Metaplastic thymoma of the mediastinum has been described (3). There were seven cases in which the spindle cell component seemed to be undergoing epithelialmesenchymal transition with loss of E-cadherin. Followup showed the lesion has a benign course. Earlier thymoma with pseudosarcomatous stroma that may simulate carcinosarcoma has been described (4). Some of these cases were misinterpreted as carcinosarcomas but follow-up suggested a benign or very low grade malignant biological behaviour. It is possible that both papers are describing the same entity, although it is difficult to be certain without the review of the histology of these lesions.

There is a separate entity of thymic epithelial/ neuroendocrine neoplasms that includes Carcinoid tumour, atypical carcinoid tumour, large cell neuroendocrine carcinomas and small cell carcinoma. These tumours can be associated with multiple endocrine neoplasia (MEN I). These tumours have local and metastatic lesions with symptoms related to paraneoplastic syndromes. Prognosis is related to tumour size, staging and surgical resection (5).

\section{Staging}

A new TNM classification of thymic epithelial tumours ( $8^{\text {th }}$ edition) was published in year 2014. The International Association for the study of lung cancer (IASLC) and the International Thymic malignancy interest group (ITMIG) assembled a large retrospective database with 10,808 patients having thymic malignancies. All thymomas are considered malignant and the capsule is induced by the tumour, hence capsule has little impact on the prognosis. The $\mathrm{T}$ component is divided into four parts, $\mathrm{T} 1$ includes both encapsulated and uncapsulated thymomas/thymic carcinomas, T1b involves extension into mediastinal pleura, T2 involves pericardium, T3 involves lung, brachiocephalic vein, superior vena cava, chest wall, phrenic nerve, hilar (extrapericardial) pulmonary vessels, T4 involves aorta, arch vessels, main pulmonary artery, myocardium, trachea or oesophagus (6-8). Recurrence is regarded as the best measure of outcome along with influence of factors including age, sex, comorbidities, and social background (9).

Radiology plays an important role in the initial evaluation of patients with thymic tumours. CT and MRI scans are helpful in characterization and staging of these tumours. Fluorodeoxyglucose positron emission tomography (FDG-PET) scan shows significant higher uptake of fluorodeoxyglucose among thymic carcinomas compared to thymomas (10).

\section{Second tumour}

Thymoma patients have increased risk of second malignancy. Follow-up data from US cancer registries point towards an elevated risk for developing B-cell non-Hodgkin lymphoma. There is also increased risk of soft tissue sarcomas (11). Cases of Kaposi sarcoma have been described in association with thymomas (12). Genetic, immunodeficiency and environmental risk factors may be at play in these cases.

\section{Thymoma in children}

Thymoma is uncommon in children but is known to occur. Patients younger than 10 years are predominately male, have advanced stage tumour and less favourable outcome. Patients present with respiratory symptoms due to a mass lesion, superior vena cava syndrome or paraneoplastic syndrome including myasthenia gravis, pure red cell aplasia, acquired hypogammaglobulinemia and connective tissue disorders (13).

\section{Canine thymoma}

Although discussion in this article is focused on human thymomas, it is interesting to note that thymoma is seen in various breeds of dogs and occurs more frequently in German Shepherd dogs. A paraneoplastic syndrome of myasthenia gravis and/or polymyositis occurs in a significant number of dogs with thymoma. Thoracic radiographs show a cranial mediastinal mass. Thymomas may be contained in a capsule and excised. Adjuvant radiation and chemotherapy may be of value in advanced cases (14).

\section{Genetics}

Familial occurrence of thymomas is well known. Nicodème et al. (15) studied a family bearing the constitutional chromosome translocation $\mathrm{t}(14 ; 20)(\mathrm{q} 24.1 ; \mathrm{p} 12.3)$. In this family 11 out of 27 patients had translocations with 3 having thymomas. Four other members had autoimmune diseases including diabetes mellitus, Grave's disease, pernicious 
anaemia, Sjogren's disease and autoimmune pancytopenia. FISH analysis showed dysregulation of RAD51L1 and/or BMP2 which may explain familial occurrence of thymomas.

There are gene expression differences between thymomas and thymic cysts. Using microarray profiling to identify changes in gene expression, it was found that 2,319 messenger RNAs were upregulated and 2,776 were down regulated in thymomas relative to thymic cysts. Large number of genes participate in cellular functions among which MHC class II protein complex assembly, assembly with peptide antigen, calcium activated phosphatidylcholine scrambling and release of cytoplasmic sequestrated NF- $\mathrm{\kappa B}$ were dysregulated (16). Pathways related to autoimmune diseases were downregulated.

A tumour related gene expression study (17) showed significantly higher thymidylate synthase in thymic carcinoma and type B3 thymoma as compared to lung cancer. The ratio of folypolyglutamyl synthase to gammaglutamyl hydrolase mRNA was significantly lower in thymic carcinoma than in B3 thymoma. Gene expression of vascular endothelial growth factor (VEGF) was higher in thymic carcinoma as compared to B3 thymoma. Epidermal growth factor receptor (EGFR) expression was significantly higher in B3 thymomas as compared to thymic carcinoma and lung cancer. However, no EGFR gene mutation was detected in these samples.

A missense mutation GTF21, chromosome 7 has been identified in indolent thymomas. This correlated better with prognosis (18). Recurrent mutations of known cancer genes including TP53, CYLD, CDKN2A, BAP1 and PBRM1 have been identified in thymic carcinomas.

Differences have been observed between thymoma associated myasthenia gravis and myasthenia gravis without a thymoma. The expression level of 169 genes showed a significant difference between the two groups with 94 upregulated and 75 down regulated. Overexpression of BTLA, CCR7, TNFRSF25, ANKRD55 was identified in thymoma tissues (19).

\section{Personalized medicine}

Pathology is at the forefront of a major change in the management of patients afflicted with neoplastic diseases. There is increasing use of molecular pathology to deliver personalized medical care. This has led to the search for molecular targets which are responsive to various antibodies and anticancer agents. Some progress has been made in targeted therapy for Thymomas and thymic carcinomas.
The PIK3/Akt/mTOR pathway plays a key role in some cancers. Some phase I/II studies have shown positive effect of mTOR inhibitors in disease control in thymomas. Akt and $\mathrm{mTOR}$ are activated in thymomas. Activation of the $\mathrm{Akt} / \mathrm{mTOR}$ pathway might participate in cell proliferation associated with tumour growth. Hence, this pathway is the most attractive target for the development of anticancer agents. Patients with mTOR inhibitor-based therapy demonstrated prolonged tumour free survival in advanced/ metastatic thymomas and thymic carcinomas (20).

Molecular analysis by next generation sequencing and single PCR based assays have shown diverse actionable mutations in thymomas. These are PIK3CA, EGFR, RET and AKT1 mutations. Heterogeneity in actionable molecular aberrations suggest that multiassay molecular profiling and individualizing treatment is the way forward in thymomas (20).

In a study (21) on molecular profiling of thymoma and thymic carcinoma, expression of ALK, HER2, HER3, MET, phospho-mTOR, p16, PDGFRA, PDGFRB, PD-L1, PTEN, and ROS1 was assessed. PDGFRA was increased in thymic carcinoma and PD-L1 in B3 thymomas and thymic carcinomas.

Protein programmed death (PD-1) and its ligand (PDL1) expression has been reported in thymic epithelial tumours. PD-L1 staining is found in epithelial cells and staining pattern is membranous and variable, please see the photographs where Figure 1 is showing most of the tumour cells staining with PD-L1 antibody (Ventana PD-L1 SP263 IHC assay staining) and Figure 2 showing absence of staining in tumour cells. Lymphocytes are staining with the antibody. There are several studies on expression of PD-1 and PD-L1 in thymic epithelial neoplasms. In one study (22) expression of PD-1 and PD-L1 was assessed by immunohistochemistry in 100 thymic epithelial neoplasms including 74 thymomas and 26 thymic carcinomas. Scoring was done by the percentage of positive T-cells or tumour cells respectively. Cases with membranous reactivity of the antibody in greater than or equal to $5 \%$ of $\mathrm{T}$ cells $(\mathrm{PD}-1)$ or tumour cells (PD-L1) were considered positive. Expression of PD- 1 was detected in 52/100 cases including 6/26 thymic carcinomas (23\%) and 46/74 thymomas (62\%). PD-L1 was positive in $61 / 100$ cases including $14 / 26$ thymic carcinomas (54\%) and $47 / 74$ thymomas (64\%). A total of $82 \%$ cases showed expression of PD-1 or PD-L1. PD-1 positive cases were associated with higher stage in thymic carcinomas $(\mathrm{P}=0.01)$ and $\mathrm{PD}-1$ negative cases with thymic carcinoma histology $(\mathrm{P}=0.0014)$. $\mathrm{PD}-\mathrm{L} 1$ positive cases were associated 

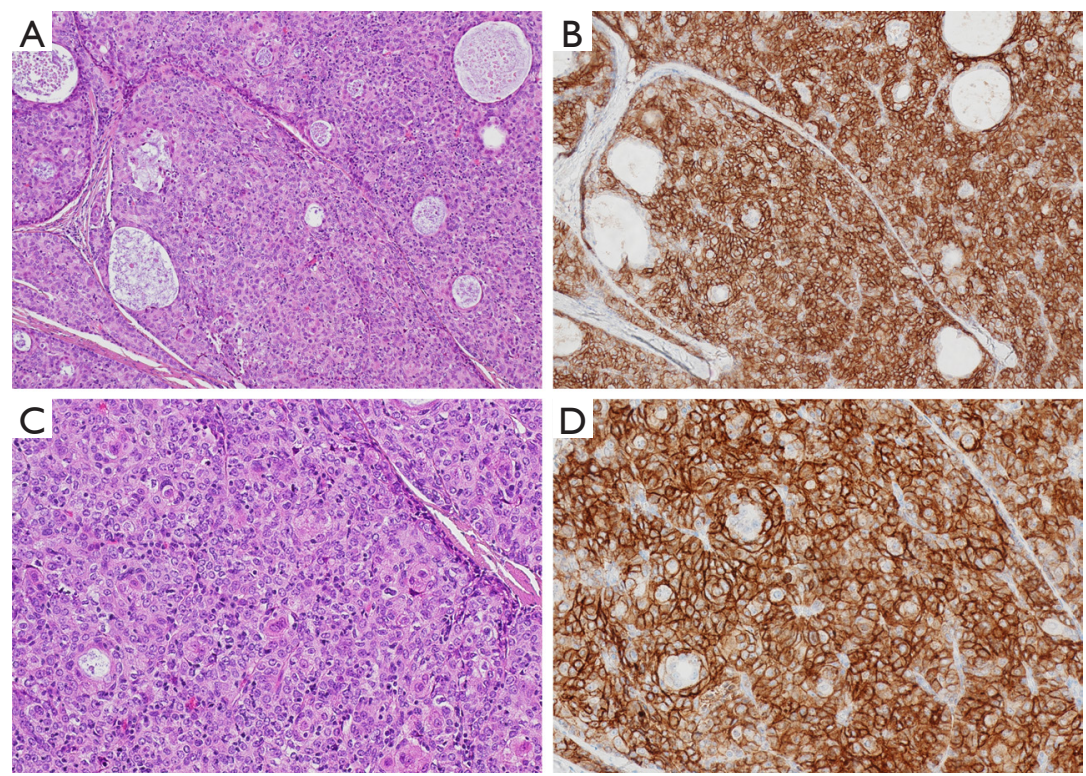

Figure 1 Thymoma (B3): complete membrane staining with PD-L1 is seen in more than $90 \%$ of tumour cells, positive for high expression (A,B: ×10, C,D: ×20; A,C: HE staining, B,D: IHC staining).
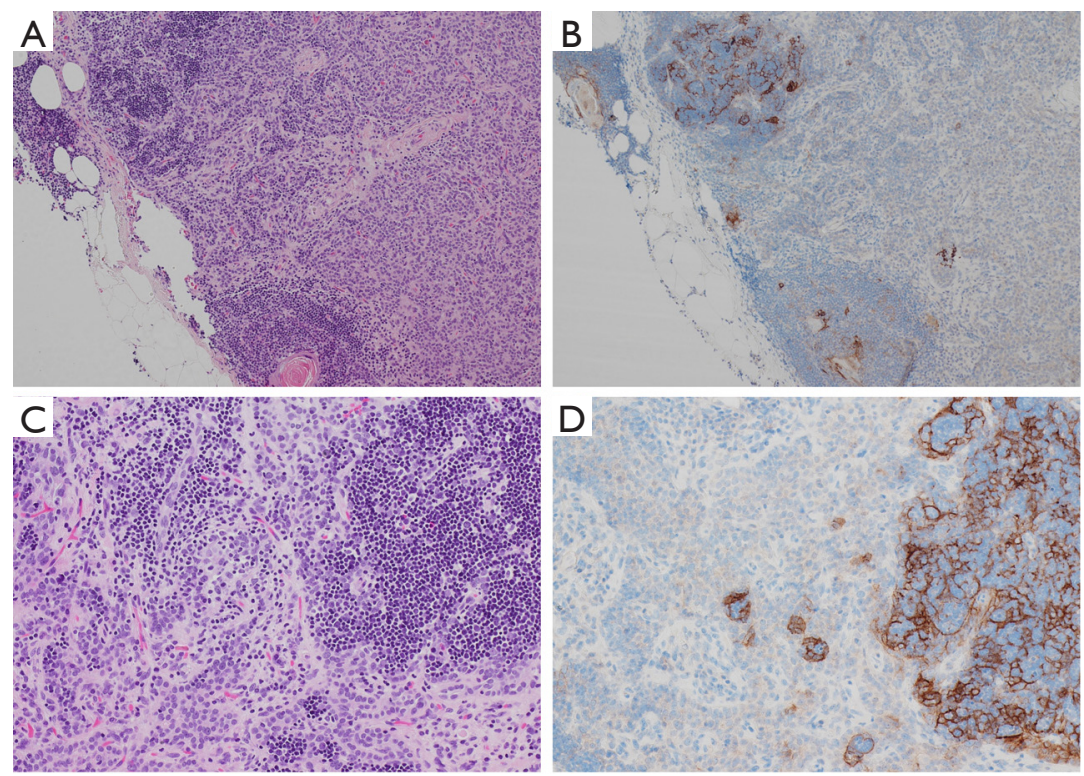

Figure 2 Thymoma (mixed B1 and B2): tumour cells are negative, while background normal thymus shows scattered positive lymphocytes with incomplete membranous staining (A,B: $\times 10, \mathrm{C}, \mathrm{D}: \times 20$; A,C: HE staining, B,D: IHC staining).

with neoadjuvant therapy in thymoma $(\mathrm{P}=0.0065)$. Study concluded that PD-1 and PD-L1 are highly expressed in thymic epithelial neoplasms raising the possibility of the use of anti-PD-1/PD-L1 therapy in treatment of these tumours. Prognostic role of these markers is uncertain and may have a role in combination with neoadjuvant treatment. Pembrolizumab has shown encouraging anti-tumour activity in patients with advanced thymic epithelial tumours. Due to severe autoimmune disorders associated with these tumours, careful monitoring is essential (23). 


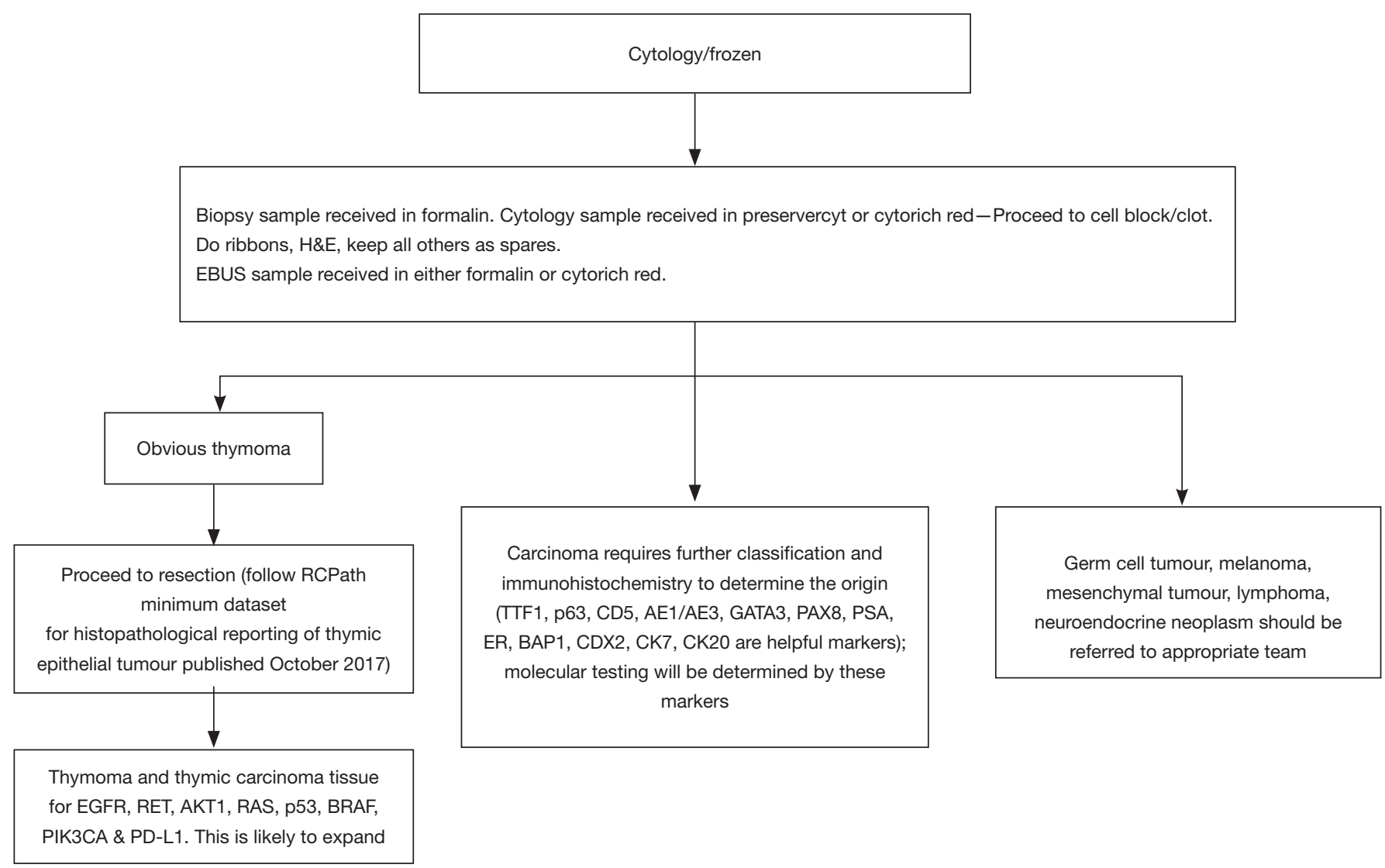

Figure 3 Protocol for histological management of a mediastinal mass. The figure shows the histopathology laboratory protocol for dealing with a biopsy from a mediastinal mass.

Efficacy and safety of the anti PD-L1 antibody in patients with relapsed and advanced thymic epithelial tumours was assessed in a phase 1 trial (24). Seven patients with thymoma and 1 patient with thymic carcinoma were enrolled in this study. There was major tumour regression in four of seven patients with recurrent thymoma treated with the anti-PD-L1 antibody, avelumab. Response was associated with immune related adverse events including myositis, myocarditis, and neuromuscular disorders. A recent article (25) has reviewed the results of clinical trials that have evaluated immunotherapy for treatment of relapsed thymoma and thymic carcinoma. Immune check point inhibitors have shown response in relapsed and refractory thymic epithelial tumours but are associated with increased risk of immune related adverse events. Paper identifies three risk reducing strategies to mitigate the risk of adverse events. In the same article there is reference to cancer vaccines such as WT1 peptide-based vaccine immunotherapy for advanced thymic epithelial neoplasms with disease stabilization in most vaccinated patients (75\%).

Overexpression of c-kit, a tyrosine kinase receptor protein encoded by protooncogene kit is described in various tumours including thymic epithelial tumours. Mutations in the kit gene have been related to response in gastrointestinal stromal tumours, myeloproliferative disorders, melanoma, and seminoma. C-kit positive cells are higher in thymic carcinomas (46\%) than in thymomas (4\%) (26). There are few case reports and a series of 11 cases of response to imatinib in unresectable thymic carcinoma where only three obtained a stable disease as best response (27). There are few drugs that have undergone evaluation in phase II trials. As $10-30 \%$ of thymomas recur even after complete resection there is need for more effective drugs targeting tumour cells, supporting stroma and vasculature supplying nutrients to neoplastic cells. New therapies require newer targets. Mutational analysis of EGFR pathway genes, RAS, EGFR, PIK3CA, AKT1\& BRAF and TP53 gene in thymic carcinoma and thymoma 
type $\mathrm{A} / \mathrm{B} 3$ has been performed in a study (28). This is one of the largest study to investigate EGFR-RAS-RAF signalling pathway in thymic carcinomas and thymoma type $\mathrm{A} / \mathrm{B} 3$. They found RAS mutations in 10 out of 54 thymic carcinomas. Other mutations included EGFR in two cases, PIK3CA in one, AKT1 in one, BRAF in none and TP53 in five cases. Among 33 thymoma cases, they found HRAS gene mutation in one, PIK3CA in two and AKT1 in one case. All these mutations were missense type activating mutations. RAS family mutations were significantly more frequent in thymic carcinomas than in thymomas type $\mathrm{A} / \mathrm{B} 3$. It seems that mutations in EGFR pathway and TP53 are more frequent in thymic carcinomas than in thymomas. Investigators have focused on the mutational status of several genes including EGFR, VEGF and IGF$1 \mathrm{R}$. Targeted drugs have been tested in selected patients with advanced thymic epithelial tumours and a positive mutational status. Targeted therapies in advanced thymic epithelial tumours appears to be promising therapeutic option in a subset of patients for which there is currently no second line treatment option available (29).

\section{Conclusions}

We are entering an era in diagnostic pathology where more is needed from less. Surgery is the treatment of choice for localized thymic tumours with complete resection being the most important prognostic factor (30). Locally advanced tumours are also best removed by surgery with post-operative chemotherapy and radiation therapy. New therapies require molecular profiling. Immune check point inhibitors appear to be an important step in achieving an improved survival in thymic epithelial tumours. For surgical management a diagnosis is required for accurate classification of mediastinal lesions. Diagnostic material obtained by minimally invasive techniques is required not only for an accurate diagnostic label but tissue is required for immunohistochemistry and molecular studies. Some tests such as PDL1 requires at least 100 tumour cells. Thymic neoplasms are no different to lung neoplasms in this respect. From a histopathological point of view a mediastinal mass should be biopsied and tissue submitted for histology. As with lung cancers it is advisable to obtain at least two cores of tissue for diagnosis, prognosis, and ancillary tests to determine the future management of these tumours. This will help surgical resection and oncological management of thymic neoplasms mainly thymomas especially recurrent thymomas with the ultimate aim of enhanced clinical outcome.

Clinical guidelines are needed to help decision making for an effective patient management strategy. I have attempted to develop an algorithm for histological management of a biopsy from the mediastinal mass (Figure 3). This is an early attempt to develop a protocol with a hope that this will be the start of a process in departments of histopathology to provide additional information to physicians treating thymic epithelial neoplasms where thoracic pathology is practiced. No doubt in future revised guidelines will be needed which are reliable, reproducible and based on evidence. These should minimise practice variation, help in decision making and serve as an indicator for external review. There is role for a scientific advisory panel composed of experts from Histopathology and oncology to firm up proposals for molecular testing in thymic epithelial neoplasms similar to lung cancers $(31,32)$.

\section{Acknowledgments}

The Author would like to acknowledge the help of Dr. Raluca Mihai Consultant Histopathologist, Nottingham University Hospitals for Photography and Immunohistochemistry Section, Histopathology Department of Nottingham University Hospitals for carrying out immunohistochemistry.

Funding: None.

\section{Footnote}

Provenance and Peer Review: This article was commissioned by the Guest Editors (Dragana Jovanovic and Semra Bilaceroglu) for the series "Thymoma" published in fournal of Thoracic Disease. The article was sent for external peer review organized by the Guest Editors and the editorial office.

Conflicts of Interest: The author has completed the ICMJE uniform disclosure form (available at: http://dx.doi. org/10.21037/jtd-2019-thym-04). The series "Thymoma" was commissioned by the editorial office without any funding or sponsorship. The author has no other conflicts of interest to declare.

Ethical Statement: The authors is accountable for all aspects of the work in ensuring that questions related to the accuracy or integrity of any part of the work are appropriately investigated and resolved. 
Open Access Statement: This is an Open Access article distributed in accordance with the Creative Commons Attribution-NonCommercial-NoDerivs 4.0 International License (CC BY-NC-ND 4.0), which permits the noncommercial replication and distribution of the article with the strict proviso that no changes or edits are made and the original work is properly cited (including links to both the formal publication through the relevant DOI and the license). See: https://creativecommons.org/licenses/by-nc-nd/4.0/.

\section{References}

1. Tateyama H, Sugiura H, Yamatani C, et al. Expression of podoplanin in thymoma: its correlation with tumour invasion, nodal metastasis and poor clinical outcome. Hum Pathol 2011;42:533-40.

2. Su XY, Wang WY, Li JN, et al. Immunohistochemical differentiation between type B3 thymomas and thymic squamous cell carcinomas. Int J Clin Exp Pathol 2015;8:5354-62.

3. Liu B, Rao Q, Zhu Y, et al. Metaplastic thymoma of the mediastinum. A clinicopathologic, immunohistochemical and genetic analysis. Am J Clin Pathol 2012;137:261-9.

4. Suster S, Moran CA, Chan JK. Thymoma with pseudosarcomatous stroma: report of an unusual histologic variant of thymic epithelial neoplasm that may simulate carcinosarcoma. Am J Surg Pathol 1997;21:1316-23.

5. Sullivan JL, Weksler B. Neuroendocrine tumours of thymus: Analysis of factors effecting survival in 254 patients. Ann Thorac Surg 2017;103:935-9.

6. Detterbeck FC, Stratton K, Giroux D, et al. The IASLC/ ITMIG thymic epithelial tumours staging project: Proposal for an evidence based stage classification system for the forthcoming (8th) edition of the TNM classification of malignant tumours. J Thorac Oncol 2014;9:S65-72.

7. Nicholson AG, Detterbeck FC, Marino M, et al. The IASLC/ITMIG thymic epithelial tumours staging project: proposals for the $\mathrm{T}$ component for the forthcoming (8th) edition of the TNM classification of malignant tumours. J Thorac Oncol 2014;9:S73-80.

8. Kondo K, Van Schil P, Detterbeck FC, et al. The IASLC/ITMIG thymic epithelial tumours staging project: proposals for the $\mathrm{N}$ and $\mathrm{M}$ components for the forthcoming (8th) edition of the TNM classification of malignant tumours. J Thorac Oncol 2014;9:S81-7

9. Fukui $\mathrm{T}$ and Yokoi $\mathrm{K}$. The new classifications for thymic epithelial tumours: benefits and problems. J Thorac Dis
2017;9:4165-8.

10. Benveniste MF, Moran C, Mawlawi O, et al. FDG PET$\mathrm{CT}$ aids in the preoperative assessment of patients with newly diagnosed thymic epithelial malignancies. J Thorac Oncol 2013;8:502-10.

11. Engels EA. Epidemiology of thymoma and associated malignancies. J Thorac Oncol 2010;5:S260-5.

12. Carvalho Araújo B, Viana Baptista S, Mascarenhas L, et al. Maxillary Sinus Kaposi Sarcoma: Case Report in an HIVNegative Patient with Thymoma. Case Rep Otolaryngol 2017;2017:3263728.

13. Liang X, Lovell MA, Capocelli KE, et al. Thymoma in children: report of 2 cases and review of the literature. Pediatr Dev Pathol 2010;13:202-8.

14. Aronsohn M. Canine thymoma. Vet Clin North Am Small Anim Pract 1985;15:755-67.

15. Nicodème F, Geffroy S, Conti M, et al. Familial occurrence of thymoma and autoimmune diseases with the constitutional translocation $\mathrm{t}(14 ; 20)(\mathrm{q} 24.1 ; \mathrm{p} 12.3)$. Genes Chromosomes Cancer 2005;44:154-60.

16. Meng FJ, Wang S, Zhang J, et al. Alteration in gene expression profiles of thymoma: Genetic differences and potential novel targets. Thorac Cancer 2019;10:1129-35.

17. Karube Y, Kobayashi S, Maeda S, et al. Tumour-related gene expression levels in thymic carcinoma and type B3 thymoma. J Cardiothorac Surg 2016;11:85.

18. Petrini I, Meltzer PS, Kim IK, et al. A specific missense mutation in GTF21 occurs at high frequency in thymic epithelial tumours. Nat Genet 2014;46:844-9.

19. Xi J, Wang L, Yan C, et al. The cancer gene atlas dataset-based analysis of aberrantly expressed genes by GeneAnalytics in thymoma associated myasthenia gravis. J Thorac Dis 2019;11:2315-23.

20. Wheler J, Hong D, Swisher SG, et al. Thymoma patients treated in a phase 1 clinic at MD Anderson Cancer Centre: response to $\mathrm{mTOR}$ inhibitors and molecular analyses. Oncotarget 2013;4:890-8.

21. Enkner F, Pichlhöfer B, Zaharie AT, et al. Molecular profiling of thymoma and thymic carcinoma: Genetic differences and potential novel therapeutic targets. Pathol Oncol Res 2017;23:551-64.

22. Weissferdt A, Fujimoto J, Moran CA, et al. Expression of PD-1 and PD-L1 in thymic epithelial neoplasms. Mod Pathol 2017;30:826-33.

23. Giaccone G, Kim C, Thompson J, et al. Pembrolizumab in patients with thymic carcinoma: a single arm, singlecentre, phase 2 study. Lancet Oncol 2018;19:347-55.

24. Rajan A, Heery CR, Thomas A, et al. Efficacy and 
tolerability of anti-programmed death-ligand 1 (PD-L1) antibody (Avelumab) treatment in advanced thymoma. J Immunother Cancer 2019;7:269.

25. Zhao C, Rajan A. Immune checkpoint inhibitors for treatment of thymic epithelial tumours: how to maximize benefit and optimize risk? Mediastinum 2019;3:35.

26. Petrini I, Zucali PA, Lee HS, et al. Expression and mutational status of c-kit in thymic epithelial tumours. J Thorac Oncol 2010;5:1447-53.

27. Salter JT, Lewis D, Yiannoutsos C, et al. Imatinib for the treatment of thymic carcinomas. J Clin Oncol 2008;26:8116.

28. Sakane T, Murase T, Okuda K, et al, A mutation analysis of the EGFR pathway genes, RAS, EGFR, PIK3CA, AKT1 and BRAF, and TP53 gene in thymic carcinoma and thymoma type A/B3. Histopathology 2019;75:755-66.

29. Marulli G, Mammana M, Rea F. Targeted therapies for the treatment of patients with advanced, recrrent or relapsed thymic epithelial tumours. Shanghai Chest 2018;2:55.

30. Kurup A, Loehrer PJ. Thymoma and thymic carcinoma: Therapeutic approaches. Clin Lung Cancer 2004;6:28-32.

31. Lindeman NI, Cagle PT, Beasley MB, et al. Molecular testing guideline for selection of lung cancer patients for EGFR and ALK tyrosine kinase inhibitors: Guideline from the College of Americal Pathologists, International association for the study of lung cancer, and Association for molecular pathology. J Thorac Oncol 2013;8:823-59.

32. Lindeman NI, Cagle PT, Aisner DL, et al. Updated molecular testing guideline for the selection of lung cancer patients for treatment with targeted tyrosine kinase inhibitors: Guideline from the College of American pathologists, the international association for the study of lung cancer, and the association for molecular pathology. Arch Pathol Lab Med 2018;142:321-46.
Cite this article as: Soomro IN. Pathology of thymomawhere are we today? J Thorac Dis 2020;12(12):7545-7552. doi: 10.21037/jtd-2019-thym-04 